\title{
The Regeneration of Axolotl Limbs Covered by Frog Skin'
}

\author{
BRUCE M. CARLSON \\ Departments of Anatomy and Biological Sciences, University of Michigan, Ann Arbor, Michigan 48109 \\ Received January 26, 1981; accepted in revised form November 23, 1981
}

\begin{abstract}
Forearm skin of: Stage XXIV Rana pipiens, which cannot regenerate limbs, was removed and placed upon the skinned forearms of young axolotls. The axolotl limbs were amputated immediately through the level of the grafts. Frog epidermis migrated to cover the amputation surface. Dedifferentiation and early blastema formation occurred beneath the frog wound epidermis. Limb regeneration continued, but in time axolotl epidermis overgrew the frog epidermis. The experiment shows that epidermis from nonregenerating frog limbs is still capable of supporting typical epimorphic regeneration.
\end{abstract}

\section{INTRODUCTION}

The limbs of most higher vertebrates are incapable of epimorphic regeneration after simple amputation. One of the principal questions regarding the loss of regenerative power in limbs is whether, during ontogeny or phylogeny, individual tissue components of limbs have lost the capacity to participate in epimorphic regeneration or whether the regenerative capacity is retained, but some coordinating function is lacking.

One strategy for investigating this question is to construct hybrid limbs, by adding one or more tissues from nonregenerating limbs onto limbs that can regenerate, and then amputate the limbs. Will the test tissue permit and/or participate in the regenerative process? In this experiment limb skin from postmetamorphic frogs, which have lost the ability to regenerate, was grafted onto the limbs of axolotls, which readily regenerate. Since skin, particularly the wound epidermis over the amputation surface, is required for any epimorphic regenerative activity (reviewed by Singer and Salpeter (1961)), the specific question was whether or not the skin from nonregenerating frog limbs could promote and maintain the regeneration of an amputated limb in the axolotl.

\section{MATERIALS AND METHODS}

Host animals were $\mathbb{1} 1 / 2$-month-old white axolotls (66to $108-\mathrm{mm}$ total length), and donor skin was taken from Stage XXIV (Taylor and Kollros, 1946) Rana pipiens with forearms approximately the same diameters as the host limbs. The frogs were obtained from the Amphibian Facility of Dr. George Nace at the University of Michigan. The axolotls were maintained in individual

\footnotetext{
${ }^{1}$ Supported by a grant from MDA.
}

plastic containers at $21^{\circ} \mathrm{C}$ and fed three times per week with beef liver.

Both axolotls and frogs were anesthetized by immersion in 1:1000 MS 222 (Sandoz). A cuff of forearm skin was removed from one (28 animals) or both (11 animals) host forearms (Fig. 1). Similar cuffs of skin were removed from the forearms of the donor frogs and sutured (with correct orientation) in place with 10-0 Perlon sutures (SSC, Switzerland). Immediately after skin grafting the axolotl limbs were amputated through the distal edge of the skin cuffs. In the animals with only one skin graft, the other limb was amputated through a similar level as a control. Immunosuppressive therapy was not used in this experiment. Eight limbs of Stage XXIV frogs were amputated at the distal forearm level to control for nonregeneration of the frog limbs.

Experimental limbs were frequently observed, and the appearance of the frog skin grafts was recorded with the aid of camera lucida. Animals were killed at numerous postoperative intervals (see Table 1), and after gross photography the limbs were fixed in Bouin's solution. All experimental and control limbs were embedded in paraffin, serially sectioned at $7 \mu \mathrm{m}$, and stained with Ehrlich's hematoxylin and eosin.

\section{RESULTS}

Controls. The amputated control limbs of the axolotls exhibited the typical regenerative reactions seen in small animals, with medium-bud to late-bud blastemas seen by $8-10$ days after amputation. The amputated frog limbs followed the expected course of nonregeneration seen in postmetamorphic anurans. In time the distal part of the limb stump was dominated by the exuberant proliferation of periosteal cartilage, the presence of a dense mat of fibrous connective tissue over the cut end 


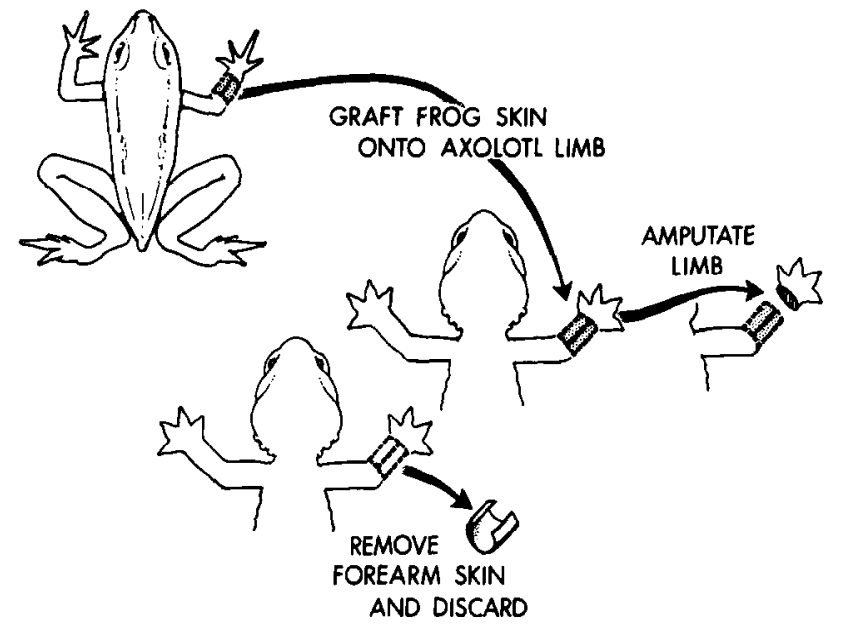

FIG. 1. Diagram of the surgical model for creating hybrid frogaxolotl limbs.

of the bone, and full thickness skin over the amputation surface (Fig. 2).

Experimental limbs. Pronounced differences in histological structure enable one to distinguish easily between frog and axolotl skin (Fig. 4). The cells and nuclei in frog epidermis are much smaller than those of the axolotl. The large Leydig cells, characteristic of axolotl epidermis, are absent in the frog. Also, frog epidermis has a tendency to desquamate in layers, a phenomenon not seen in the axolotl. Frog dermis contains prominent mucous glands of varying sizes, depending upon the age of the animal and the location. The bottom layer of dermis consists of a very dense layer of orthogonally layered collagen fibers. Axolotl dermis does not contain glands, and the dense collagenous basal layer is less prominent than that of the frog.

After amputation, the wound surface consistently became healed over by frog epidermis. The frog wound epidermis became well integrated with the underlying axolotl tissues, and by 4 to 5 days after amputation a typical dedifferentiative reaction was seen in the distal part of the deep tissues of the hybrid limb stumps (Fig.
3). In no case did the frog dermis appear to participate in the dedifferentiative reaction. The frog epidermis was thickened over areas of damaged mesoderm or early accumulations of blastemal cells in a manner resembling the apical cap that forms from axolotl epidermis. Of 15 limbs at appropriate stages, 11 possessed areas of thickened apical epidermis that contained from 2 to 30 more layers of epidermal cells than the lateral epidermis. The apical thickenings of frog epidermis were, however, not so prominent as those seen in normal axolotl epidermis.

In limbs that remained completely covered by frog epidermis, a blastema typical of that seen in normal axolotl limbs formed midway through the second week (Fig. 5). At later stages the number of limbs that remained covered by pure frog epidermis decreased considerably because of overgrowth and replacement of the frog epidermis by axolotl epidermis (Table 1). One hybrid regenerate, sampled at 16 days after amputation, had advanced to a stage at which three digital primordia were present (Figs. 6 and 7). Because most regenerates over 15 days old were either partially or completely covered by axolotl epidermis, it was not possible to determine whether pure frog epidermis could support morphologically normal regencration beyond the formation of early digital primordia, as indicated in Fig. 7.

An intense cellular immune response, directed against the tissues of the graft (Taban and Connelly, 1972), was not seen in these animals. Superficial layers of the epidermis of the graft underwent desquamation, and proximal axolotl epidermis began to overgrow the frog epidermis (Fig. 8) in a manner similar to that described by Taban and Connelly (1972, Fig. 9) in newt to axolotl grafts. Relatively few lymphocytes invaded the dermal component of the grafts, but after the first week, increasing numbers of phagocytic cells with ingested cell debris, including pigment, were seen in the frog dermis.

The dermal component of the frog skin grafts underwent a gradual involution, with no evidence that cellular components of the dermis participated in the re-

FIG. 2. Forearm of control frog 20 days after amputation. The amputation surface is by this time covered with full thickness skin. Between the distal ends of the amputated bones and the skin is a layer of dense fibrocellular material (F). Subperiosteal cartilage (C) has formed around the bones as a response to amputation. Hematoxylin and eosin. $43 \times$.

FIG. 3. Frog-axolotl hybrid limb 5 days after amputation. The amputation surface has been covered by frog epidermis, and osteoclastic erosion of bone and dedifferentiative changes in the soft tissues are occurring in the distal part of the limb stump. The junction between frog and axolotl skin is indicated by an asterisk at the right. The junction at the left (enclosed by rectangle) is shown at higher magnification in Fig. 4. The distal (retracted on the right) border of frog dermis is marked with arrows. It remains sharp in all limbs. Hexatoxylin and eosin. $42 \times$.

FIG. 4. Higher power of view of the junction between frog and axolotl skin on the limb seen in Fig. 3. Proximal axolotl epidermis (A) is beginning to overgrow the graft of frog skin. C-Basal layer of connective tissue in frog dermis; F-frog epidermis; G-glands in frog dermis. Hematoxylin and eosin. $132 \times$.

FIG. 5. Eleven-day regenerate of a hybrid limb showing a well-developed regeneration blastema (B) completely covered by frog epidermis. The junctions between frog and axolotl epidermis are indicated by arrows. Hematoxylin and eosin. $31 \times$. 

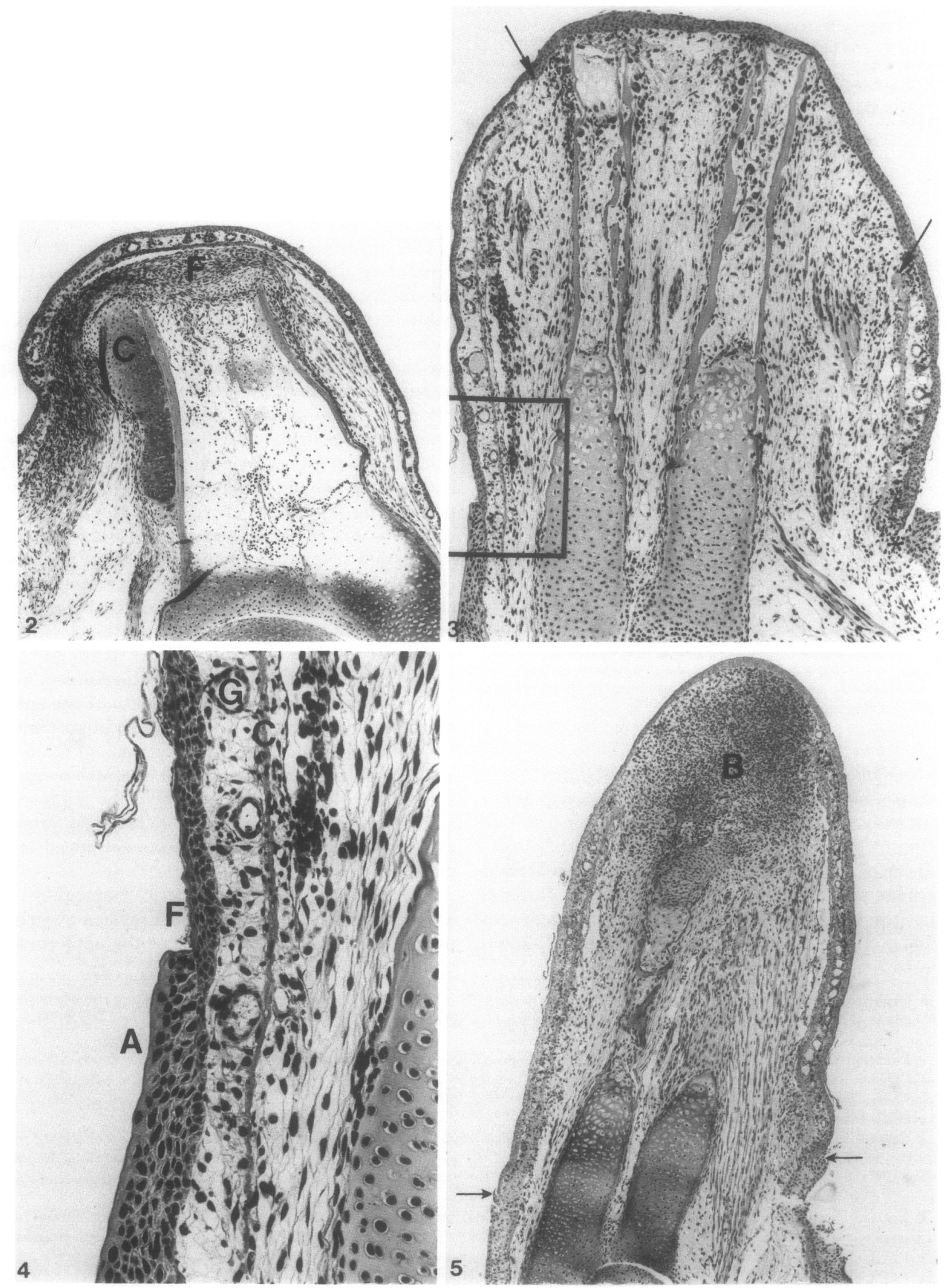
TABLE 1

DEGREE OF OVERGROWTH OF EXPERIMENTAL LIMBS BY AXOLOTL EPIDERMIS

\begin{tabular}{cc}
$\begin{array}{c}\text { Days after } \\
\text { amputation }\end{array}$ & $\begin{array}{c}\text { Degree of overgrowth } \\
\text { by axolotl epidermis }\end{array}$ \\
\hline 1 & 0 \\
2 & 0 \\
3 & 0 \\
4 & 0 \\
5 & 0 \\
6 & $0,0,1$ \\
7 & 1 \\
8 & 0 \\
9 & $2,2,3$ \\
10 & 1 \\
11 & $0,0,1,1,1$ \\
12 & 0 \\
14 & $0,1,2,3$ \\
15 & 2 \\
16 & $0,2,2$ \\
18 & $2,2,3,3$ \\
19 & 3 \\
20 & $2,2,3$ \\
21 & 2,2 \\
24 & 3,3 \\
28 & 3 \\
29 & 3 \\
44 & $3,3,3$ \\
\hline
\end{tabular}

Note. 0-No overgrowth by axolotl epidermis; 1 -axolotl epidermis overgrowing the frog skin cuffs, but not present over the wound surface; 2-axolotl epidermis overgrowing the amputation surface, but some frog epidermis remaining; 3 -complete overgrowth by axolotl epidermis, no frog epidermis remaining.

generation blastema, but the greater variety of cell sizes and shapes of the deep tissues in the axolotl made it difficult to exclude definitively any cellular contribution from the frog dermis. Nevertheless, the dense collagenous layer of frog dermis persisted in the regenerates throughout the course of these experiments. In older regenerates the dense dermal connective tissue was found deep within the substance of the limb, and axolotl cells comprising the regenerate proper were located beneath and outside this layer. There was no evidence that the persisting sheet of frog dermal connective tissue interfered with morphogenesis; in fact, in two cases forearm bones differentiated around portions of persisting dermis, causing the dermal remnants to be completely surrounded by a cartilaginous matrix (Fig. 9).

\section{DISCUSSION}

This experiment shows that epidermis from nonregenerating limbs (Stage XXIV) of the frog (1) retains the ability to support limb regeneration and (2) is capable of supporting regeneration in urodeles. On the basis of the data collected in this experiment, one can say that limb epidermis from this stage frog is capable of supporting dedifferentiation and growth (through the appearance of early digital primordia) of the regeneration blastema. Because in no case did the frog epidermis withstand overgrowth by epidermis of the axolotl long enough for complete digit formation, it is not possible to state whether or not the frog epidermis could support complete and normal morphogenesis.

It is now clear that the decline and ultimate loss of regenerative capacity in Rana pipiens are not due to a change in the capacity of the epidermal component to participate in the process, but can rather be attributed to changes in the underlying tissues. It would be useful to perform similar hybridization experiments with the skin of older frogs to see if the ability of the epidermis to support regeneration declines with increasing age of the donor. The fact that the requirement for a wound epidermis in regeneration is not species or even order specific is not surprising in view of the many embryological experiments in which hybrids of interacting components from two species have developed into normal structures.

The question of morphogenetic "neutrality" of the epidermis remains an open one. Previous experimental data (Carlson, 1975) suggest that the epidermis has a

FIG. 6. Gross photograph of a 16-day regenerate of a hybrid limb. The cuff of frog skin is pigmented, in contrast to the white skin of the host. The plane of amputation is indicated by arrows. Early digital condensations are visible in histological sections (see Fig. 7). Hematoxylin and eosin.

Fig. 7. Parasagittal section from the 16-day regenerate shown in Fig. 6. The forearm and regenerate remain completely covered by frog epidermis. The junctions between frog and axolotl epidermis are indicated by arrows. Within the blastema skeletal primordia are taking shape and early digital condensations (D) have formed. Because of an obliquity of the regenerate only one digital primordium can be seen in this section. Hematoxylin and eosin.

FIG. 8. Section through a hybrid axolotl limb 6 days after amputation. This section shows the extensive migration of axolotl epidermis (A) over the proximal part of the cuff of frog skin. The bottom of the photomicrograph is proximal and the top is distal on the limb. Note the absence of a strong cellular immune reaction directed against the grafted frog skin. C-Basal layer of connective tissue in frog dermis; F-frog epidermis. Hematoxylin and eosin. $79 \times$.

FIG. 9. Section through the zeugopodial region of a 25-day hybrid limb showing the differentiation of cartilagenous skeletal elements around persisting connective tissue elements of frog dermis (arrows). The dark cells in this section are pigment-laden phagocytic cells associated with the late stages of removal of frog dermis. This regenerate is completely covered by axolotl epidermis. The lower edge of the figure is proximal, and the upper edge is distal. R-radius; U-ulna. Hematoxylin and eosin. 53×. 

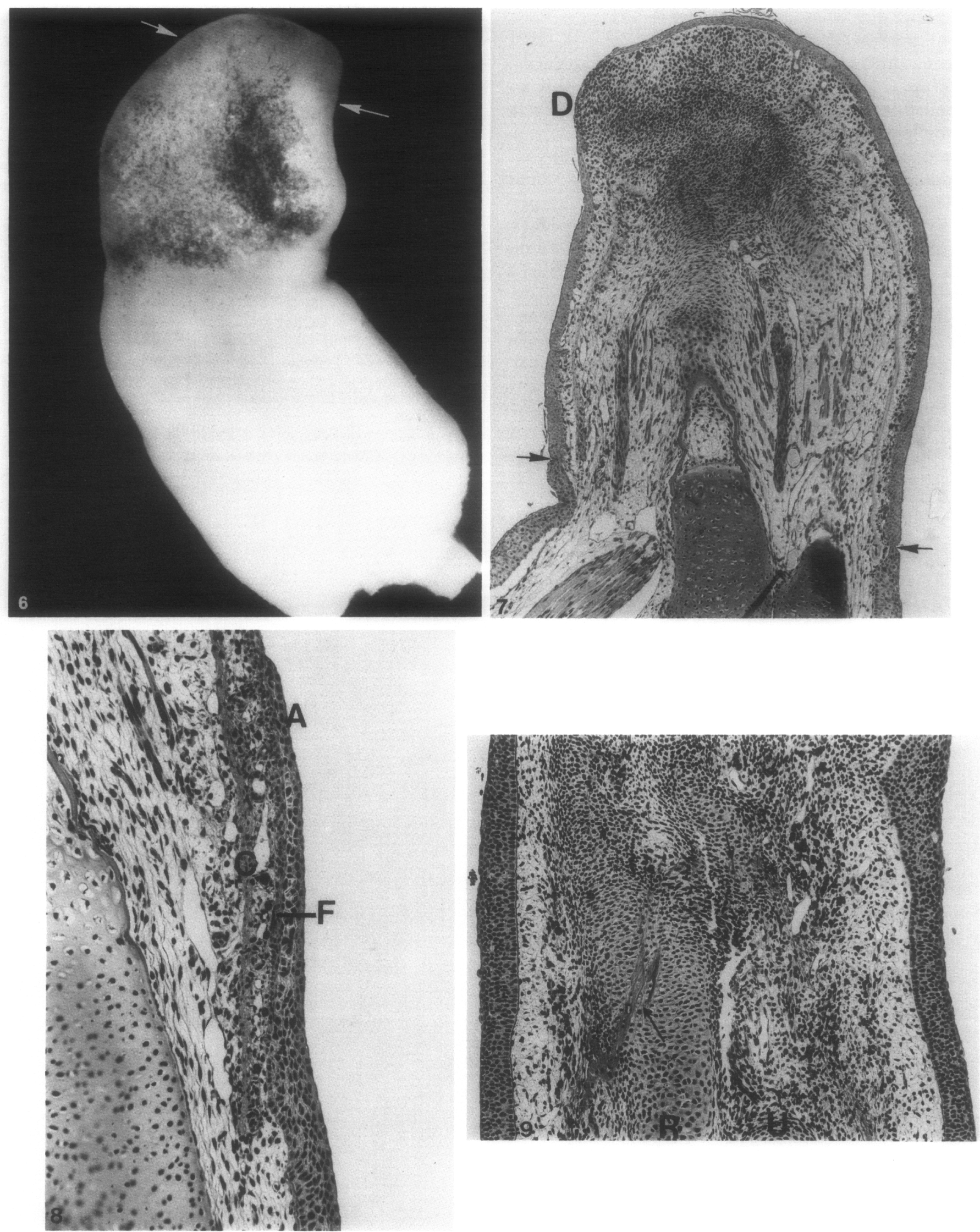
neutral quality, at least along the transverse axes. Some recent models of limb morphogenesis (Maden, 1977; Stocum, 1978) assign a zero value to the limb epidermis in terms of positional values, but implicit in this assignment is the ability to interact with underlying cells containing positive positional values. If the epidermis were truly neutral, then one would expect that any epithelial covering would suffice. In contrast, the wound epidermis could play a more specific role, whether permissive or instructive, that involves at least some form of recognition between the epidermal and underlying cells. The degree to which there is linkage between a possible role of the epidermis in the initiation of regeneration and in morphogenesis remains to be seen.

It was originally hoped that the differences in size and structure between frog and axolotl cells might permit the effective use of hybrid limbs constructed with internal tissues of frog limbs for tracing studies. However, the reactions of frog dermis in this experiment plus the reactions of implants of frog muscle (unpub- lished experiments) did not support the thought that this could prove to be a general model for systematically investigating the regenerative properties of frog tissues. In my experience this hybrid model is useful principally for testing the properties of foreign epidermis.

\section{REFERENCES}

CARLSON, B. M. (1975). The effects of rotation and positional change of stump tissues upon morphogenesis of the regenerating axolotl limbs. Develop. Biol. 47, 269-291.

MADEN, M. (1977). The regeneration of positional information in the amphibian limb. J. Theor. Biol. 69, 735-753.

SINGER, M., and SALPETER, M. M. (1961). Regeneration in vertebrates: the role of the wound epithelium. In "Growth in Living System" (M. X. Zarrow, ed.), pp. 277-311. Basic Books, New York.

STOCUM, D. L. (1978). Organization of the morphogenetic field in regenerating amphibian limbs. Amer. Zool 18, 883-896.

TABAN, C. H., and CONNELLY, T. G. (1972). Histological observations on allografts and reciprocal xenografts in newts (Notophthalmus viridescens) and axolotls (Ambystoma mexicanum). J. Exp. Zool. 182, 15-30.

TAYlor, A. C., and Kollros, J. J. (1964). Stages in the normal development of Rana pipiens larvae. Anat. Rec. 94, 7-23. 